\title{
Which Patients With a Unilateral Hearing Aid for Symmetric Sensorineural Hearing Loss Have Auditory Deprivation?
}

\author{
Hyun Jin Lee ${ }^{1,2}$ (D) $\cdot$ Jeon Mi Lee ${ }^{3}$ (D) $\cdot$ Gina Na ${ }^{1}$ (D) $\cdot$ Young Min Moon ${ }^{1}$ (D) $\cdot$ Chan Lee $^{1}$ (D) $\cdot$ Jinsei Jung $^{1}$ (D) \\ ${ }^{I}$ Department of Otorhinolaryngology, Yonsei University College of Medicine, Seoul; ${ }^{2}$ Department of Otorhinolaryngology-Head and Neck \\ Surgery, Incheon St. Mary's Hospital, College of Medicine, The Catholic University of Korea, Seoul; ${ }^{3}$ Department of Otorhinolaryngology, \\ Ilsan Paik Hospital, Inje University College of Medicine, Goyang, Korea
}

Objectives. The aim of study is to find conditions that aggravate auditory deprivation in patients with symmetric hearing loss after unilateral digital, non-linear hearing aid (HA).

Methods. In the retrospective case-comparison study, we assessed 47 patients with symmetric sensorineural hearing loss (SNHL), wearing unilateral conventional HAs. Audiological outcomes were assessed $>1$ year after HA fitting (mean duration, 31.0 months). Pure-tone audiometry in HA-aided and HA-unaided conditions was performed over time. Word recognition score (WRS) was evaluated at the most comfortable listening level.

Results. The initial pure tone average of four frequency thresholds at 500,1,000, 2,000, and 4,000 $\mathrm{Hz}\left(\mathrm{PTA}_{4}\right)$ did not show a difference of $>5 \mathrm{~dB}$ HL between HA-aided and HA-unaided ears. WRS progressively decreased for both HA-aided and HA-unaided ears although the extent of decrease was significantly greater for HA-unaided (7.6\%) than for HAaided ears $(5.1 \%, P<0.05)$. Notably, auditory deprivation in HA-unaided ears was significantly greater in patients with an initial $\mathrm{PTA}_{4} \geq 53 \mathrm{~dB} \mathrm{HL}(P<0.001)$.

Conclusion. Bilateral HAs are strongly recommended, particularly for patients with moderate to severe SNHL to prevent auditory deprivation in the contralateral ear.

Keywords. Auditory Deprivation; Sensorineural Hearing Loss; Unilateral Hearing Aid; Speech Intelligibility

\section{INTRODUCTION}

Sensorineural hearing loss (SNHL) is one of the most common problems in the elderly [1]. A conventional hearing aid (HA) is considered as the first treatment for SNHL [2]. For patients with symmetric SNHL, bilateral HAs are recommended to achieve the acoustic benefits from binaural hearing [3]. However, persuading patients with symmetric SNHL to receive bilateral HAs remains a challenge because of cost and convenience issues.

\footnotetext{
- Received March 18, 2019

Revised May 29, 2019

Accepted June 7, 2019

- Corresponding author: Jinsei Jung

Department of Otorhinolaryngology, Yonsei University College of Medicine,

50-1 Yonsei-ro, Seodaemun-gu, Seoul 03722, Korea

Tel: +82-2-2228-3603, Fax: +82-2-393-0580

E-mail: JSJUNG@yuhs.ac
}

Several studies have reported a correlation between auditory deprivation and amplification by HA. In adults with asymmetric SNHL, an improvement in suprathreshold speech recognition scores was observed for the affected ear by hearing amplification, which indicated resolution of the auditory deprivation [4]. In the studies that compared individuals with unilateral HA and those with bilateral HA, the interaural difference in the word recognition score (WRS) was relatively more preserved in the latter, indicating that HAs can prevent auditory deprivation $[5,6]$.

The test for speech intelligibility generally involves evaluation of the optimum performance for phonetically balanced word recognition at the most comfortable listening level. This optimum performance is also known as the phonetically balanced maximum (PBmax) [7], which is determined by both peripheral and central auditory processing. Nevertheless, PBmax could be adjusted after HA use because a properly fitted HA increases

Copyright (C) 2020 by Korean Society of Otorhinolaryngology-Head and Neck Surgery.

This is an open-access article distributed under the terms of the Creative Commons Attribution Non-Commercial License (https://creativecommons.org/licenses/by-nc/4.0) which permits unrestricted non-commercial use, distribution, and reproduction in any medium, provided the original work is properly cited. 
the audibility of parts of the affected frequency range and, thus, enables more information to be presented for central auditory processing. In addition, HAs can stabilize speech intelligibility, as confirmed by the findings of previous studies that demonstrated a greater decrease in PBmax for HA-unaided ears than for HA-aided ears $[4,8]$. However, conditions that aggravate auditory deprivation in patients with symmetric SNHL have not been deeply evaluated yet.

Meanwhile, digital signal processing enables complex processing beyond what is possible with analog aids with nonlinear processing, noise reduction, speech enhancement, and feedback reduction [9]. Additionally, the NAL-NL2 fitting program helps to achieve better speech intelligibility and overall loudness comfort [10]. However, we are unsure as to whether these advantages with the nonlinear and digital HAs actually affected auditory deprivation in the unilateral HA fitting condition or not.

Therefore, in the present study, we compared the changes in WRS between the HA-aided ear and the HA-unaided ear in patients with symmetric SNHL who received a unilateral digital signal processing and nonlinear HA. We also analyzed subgroups that were more susceptible to auditory deprivation after unilateral HA insertion.

\section{MATERIALS AND METHODS}

\section{Subjects}

Between 2001 and 2016, patients who received conventional HAs at the Department of Otorhinolaryngology in Severance Hospital, Seoul, Korea were retrospectively reviewed. We set our standard to recruit patients with strict criteria. First, we selected patients with symmetric SNHL ( $<10 \mathrm{~dB}$ difference) who were fitted with only a unilateral HA (ipsilateral ear), though the majority of patients with symmetric hearing loss had bilateral HA fittings. Second, we selected patients who used their HA for $>1$ year and 8 hours every day until the last visit. From these strict criteria for the enrollment, only 47 adults with symmetric SNHL who received a unilateral HA were retrospectively recruited for this study.

The included patients were aged $25-82$ years (mean \pm standard

\section{H I G H L I G H T S}

- Auditory deprivation in the unaided ear is observed in patients who have symmetric sensorineural hearing loss and fit unilateral hearing aid.

- Auditory deprivation in the unaided ear is more severe in patients with poor auditory threshold.

- Patients with mild to moderate hearing loss rarely show auditory deprivation in the unaided ear even after unilateral hearing aid fitting. deviation [SD], $70.0 \pm 13.8$ years) and comprised 25 men and 22 women. The mean follow-up period was $31.0 \pm 26.2$ months (range, 12 to 108 months) from the date of HA insertion. All types of hearing loss in the participants were categorized as SNHL. All participants had used their HAs for $>1$ year with the acclimatization time of 2 months at least. Twelve patients received HA in the right ear, while the remaining received HA in the left ear.We prescribed and performed the computed tomography (CT) scan only ife middle ear problems were suspected. CT showed the absence of pathological lesions in the middle ear or mastoid that could affect hearing conditions. All patients showed symmetric hearing loss, with air-bone gaps of $<10 \mathrm{~dB}$ $\mathrm{HL}$ across all frequencies. Differences in the hearing threshold between the two ears were $<10 \mathrm{~dB}$ HL. Following HA insertion, the change in the unaided hearing level was $<5 \mathrm{~dB} H \mathrm{HL}$ (Table 1). We evaluated the average of aided pure tone thresholds at the very last session for each patient.

All patients used the digital, nonlinear type of HAs. The HA fitting program was NAL-NL2, which is the second-generation prescription procedure from The National Acoustic Laboratories for fitting wide dynamic range compression instruments. HA fitting for patients aimed at making good speech intelligible and overall loudness comfortable. We also fitted patients with real ear measurement to get more precise insertion gain. The types of HA used varied from an open-fit, receiver in the ear (RIC) type to a closed fit, complete in-ear type, depending on the patient's preference and the condition of the external auditory canal. Proprietary signal processing techniques such as feedback cancellation and active noise cancellation were applied to all patients. In cases of RIC type, directional microphones were chosen. This study was approved by the local Ethics Committee of Yonsei University (No. 4-2017-0421). Since this is a retrospec-

Table 1. Subject demographic data

\begin{tabular}{|c|c|}
\hline Characteristics & Value $(n=47)$ \\
\hline Age at the fitting $\mathrm{HA}(\mathrm{yr})$ & $70.0 \pm 13.8$ \\
\hline \multicolumn{2}{|l|}{ Sex } \\
\hline Male & $25(53.1)$ \\
\hline Female & $22(46.8)$ \\
\hline \multicolumn{2}{|l|}{ Side of wearing $\mathrm{HA}$} \\
\hline Right & $33(70.2)$ \\
\hline Left & $14(29.8)$ \\
\hline \multicolumn{2}{|c|}{$\mathrm{PTA}_{4}$ in HA-fitted ear ( $\mathrm{dB} \mathrm{HL}$ ) } \\
\hline Initial PTA 4 & $53.6 \pm 12.4$ \\
\hline Last PTA 4 & $55.8 \pm 11.5$ \\
\hline \multicolumn{2}{|c|}{$\mathrm{PTA}_{4}$ in unaided ear $(\mathrm{dB} \mathrm{HL})$} \\
\hline Initial PTA 4 & $49.4 \pm 13.9$ \\
\hline Last PTA 4 & $53.0 \pm 13.2$ \\
\hline \multicolumn{2}{|c|}{ Average of aided PTA $\mathrm{P}_{4}(\mathrm{~dB} \mathrm{HL})$} \\
\hline HA-fitted ear & $44.4 \pm 8.4$ \\
\hline
\end{tabular}

Values are presented as mean \pm standard deviation or number (\%). $\mathrm{HA}$, hearing aid; $\mathrm{PTA}_{4}$, pure tone average of four frequency thresholds $(0.5,1,2$, and $4 \mathrm{kHz})$. 
tive study, no informed consent was obtained.

\section{Audiological analysis}

Audiological evaluations, including pure-tone audiometry and speech audiometry, were performed before and more than 1 year after HA insertion for the HA-aided and HA-unaided ears. The most recent data obtained for each patient were used for statistical analyses. The pure-tone air (250-4,000 Hz) and bone conduction $(250-4,000 \mathrm{~Hz})$ thresholds were measured using clinical audiometers in a double-walled audio booth. The mean pure-tone audiometry thresholds for air conduction at $0.5,1,2$, and $4 \mathrm{kHz}$ $\left[\mathrm{PTA}_{4}=(\right.$ threshold at $\left.0.5 \mathrm{kHz}+1 \mathrm{kHz}+2 \mathrm{kHz}+4 \mathrm{kHz}) / 4\right]$ were calculated.

The speech recognition threshold was defined as the level at which patients heard and correctly repeated spondaic words $50 \%$ of the time. A spondee has two Korean syllables, with equal stress placed on each syllable. A word recognition test was performed to obtain the maximal WRS, which were measured at the most comfortable hearing level using 50 monosyllabic Korean words that are heard during everyday life. The Korean words were from a validated and standardized resource [11] and were phonetically balanced.

\section{Statistical analysis}

All statistical analyses were performed using IBM SPSS ver. 21.0 (IBM Corp., Armonk, NY, USA). The results of multiple experiments are presented as mean \pm SD. Continuous variables were compared using Mann-Whitney test and Wilcoxon signedrank test for evaluating differences between unpaired and paired groups, respectively. Multiple regression analysis was performed with stepwise selection using a general linear model. For all statistical tests, a $P$-value $<0.05$ was considered to be statistically significant.

\section{RESULTS}

\section{Comparison of the hearing threshold and WRS between HA-} aided and $\mathrm{HA}$-unaided ears

The initial $\mathrm{PTA}_{4}$ (before HA insertion) was 53.6 $\pm 12.4 \mathrm{~dB} \mathrm{HL}$
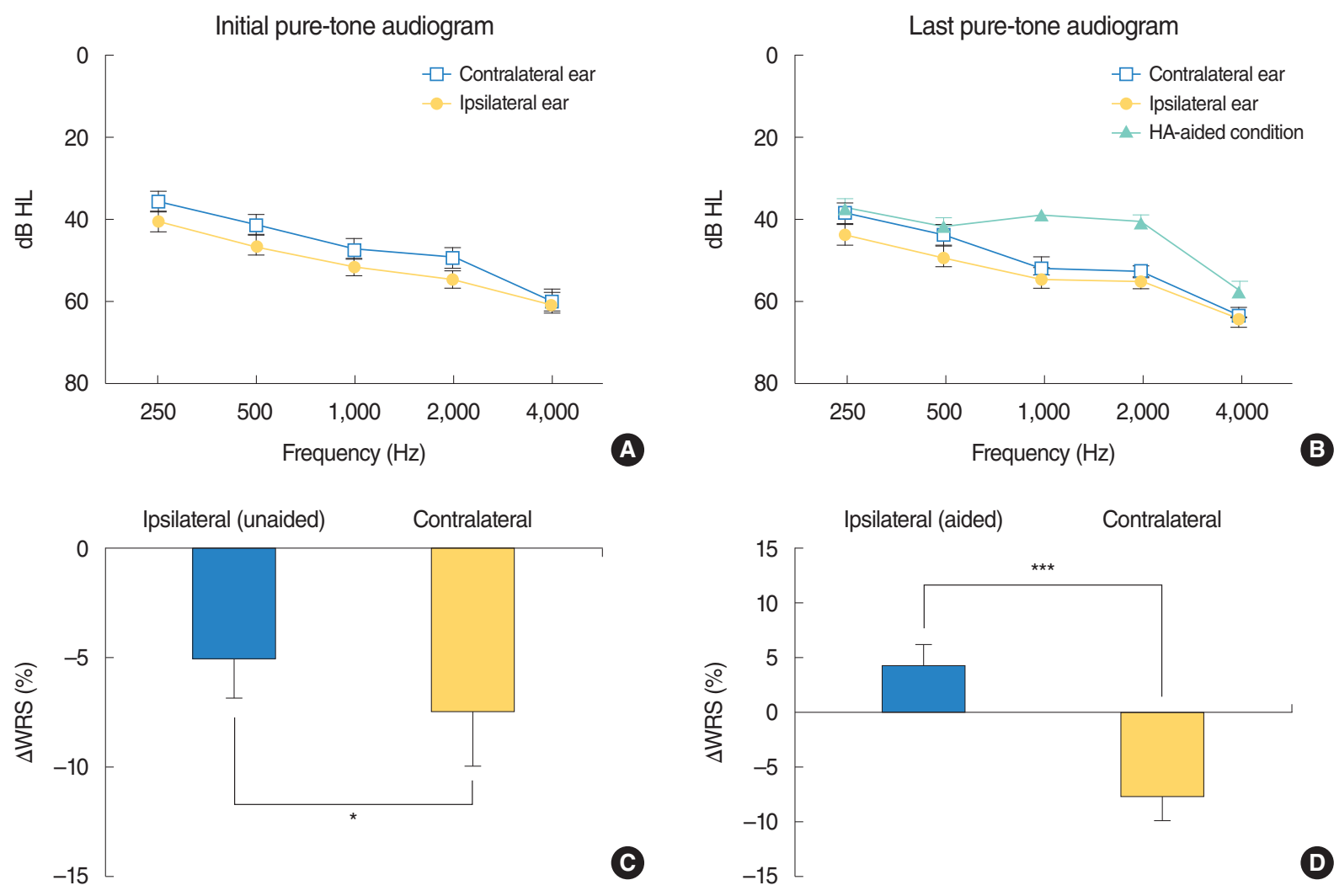

Fig. 1. Pure-tone audiometry and word recognition test for the ear fitted with a hearing aid $(\mathrm{HA})$ and the contralateral unaided ear in a patient with symmetric sensorineural hearing loss. (A) Initial unaided air-conduction audiometry is performed for the HA-aided (ipsilateral) and the HAunaided (contralateral) ears. (B) The last unaided air-conduction audiometry session is performed for the ipsilateral and the contralateral ears. Aided air-conduction audiometry for the ipsilateral ear is also performed via a sound field test using a loudspeaker. (C) The ipsilateral ear (unaided condition) shows a significantly smaller decrease in the word recognition score (WRS) than the contralateral ear. $\triangle W R S$ indicates difference in the WRS between the first and last tests. (D) $\triangle \mathrm{WRS}$ in the ipsilateral ear (aided condition) more significantly differs from that in the contralateral ear. ${ }^{\star} P<0.05,{ }^{\star \star \star} P<0.001$. 
for the ipsilateral ears and $49.4 \pm 13.9 \mathrm{~dB}$ HL for the contralateral ears; this indicated symmetric hearing thresholds in both ears (Fig. 1A). Even in the last pure-tone analysis after HA insertion, the symmetric thresholds obtained in pure-tone audiograms did not change (Fig. 1B). The most recently derived $\mathrm{PTA}_{4}$ was $55.8 \pm$ $11.5 \mathrm{~dB}$ for the ipsilateral ear and $53.0 \pm 13.2 \mathrm{~dB}$ in the contralateral ear. Thresholds did not deteriorate by more than $5 \mathrm{~dB}$ when compared with the initial hearing thresholds on each side.

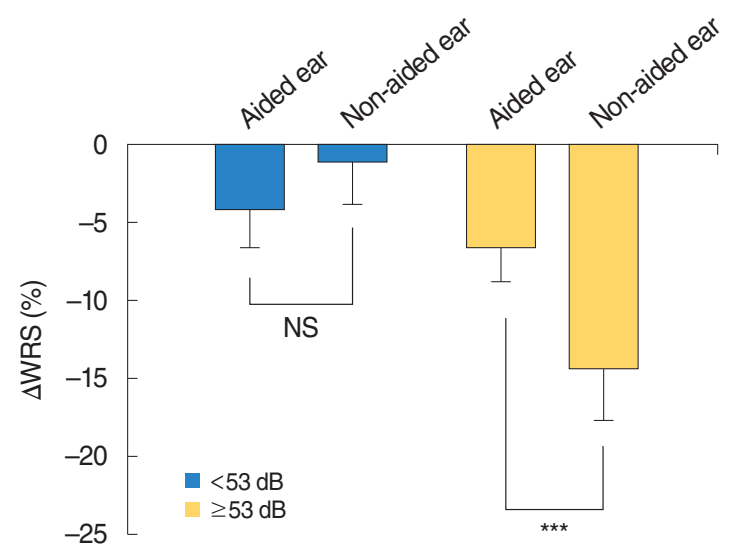

Fig. 2. Assessment of the decrease in the word recognition score (WRS) depending on the initial hearing threshold in patients with symmetric sensorineural hearing loss who had worn a unilateral hearing aid for more than 1 year. Patients are classified according to a mean pure-tone audiometry thresholds $\left(\mathrm{PTA}_{4}\right)$ of $<53$ or $\geq 53 \mathrm{~dB} \mathrm{HL}$. There is no difference in $\triangle W R S$ between the aided ear and non-aided ear in patients with an initial hearing threshold of $<53 \mathrm{~dB} \mathrm{HL}$. However, in patients with an initial hearing threshold of $\geq 53 \mathrm{~dB} \mathrm{HL}$, the decrease in WRS for the non-aided ear is significantly larger than that for the aided ear. PTA 4 , the average threshold at 500, 1,000, 2,000, and $4,000 \mathrm{~Hz} ; \triangle W R S$, difference in the WRS between the first and last tests; NS, not significant. ${ }^{\star \star *} P<0.001$.

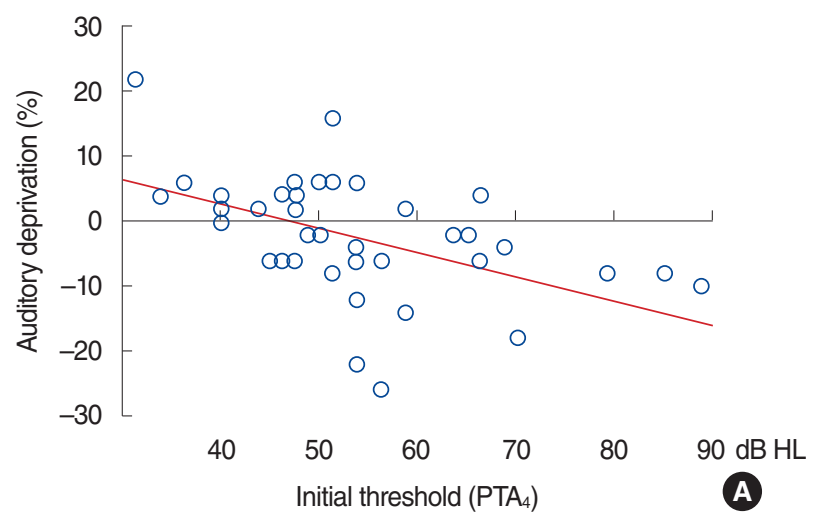

The most recent HA-aided $\mathrm{PTA}_{4}$ for the ipsilateral ear was $44.4 \pm$ $8.4 \mathrm{~dB}$ (Fig. 1B). Specifically, the hearing gains at 1,000 and $2,000 \mathrm{~Hz}$ were approximately $20 \mathrm{~dB} \mathrm{HL}$, indicating that HA insertion was reasonable. Subsequently, we analyzed changes in WRS ( $\triangle \mathrm{WRS}$ ) in both ears. WRS decreased by $5.1 \% \pm 12.5 \%$ in the ipsilateral ears $7.6 \% \pm 16.5 \%$ in the contralateral ears; the difference between ears was statistically significant $(P<0.05)$ (Fig. 1C). When we compared WRS in HA-aided ipsilateral ear and unaided contralateral ear, the difference in change of WRS was more significant between two ears $(P<0.001)$ (Fig. 1D).

\section{Effects of the initial hearing threshold on auditory deprivation in the HA-unaided ear}

We also investigated whether the initial mean pure-tone audiometry thresholds affected the severity of auditory deprivation in the unaided ear. To compare patients with good and with poor initial hearing thresholds, we classified the patients according to $\mathrm{PTA}_{4} \geq 53 \mathrm{~dB} \mathrm{HL}$ (poor hearing threshold) or $<53 \mathrm{~dB}$ $\mathrm{HL}$ (good hearing threshold). The cutoff value of $53 \mathrm{~dB} H \mathrm{HL}$ was determined by analyzing receiver operating characteristic curve for predicting auditory deprivation with the best sensitivity and specificity, where auditory deprivation in unaided ear was defined as more than $5 \%$ of difference in $\triangle \mathrm{WRS}$ between the HAaided and HA-unaided ears. As a result, no significant difference in $\triangle$ WRS between HA-aided and unaided ears was observed in the group with good initial hearing thresholds, whereas a marked decrease of $\triangle \mathrm{WRS}$ was observed in the group with a poor initial hearing threshold (Fig. 2).

In addition, the initial threshold in $\mathrm{PTA}_{4}$ was strongly correlated with the difference in $\triangle \mathrm{WRS}$ between the ipsilateral and contralateral ears, which was denoted as auditory deprivation in Fig. 3A. Auditory deprivation increased as the initial hearing

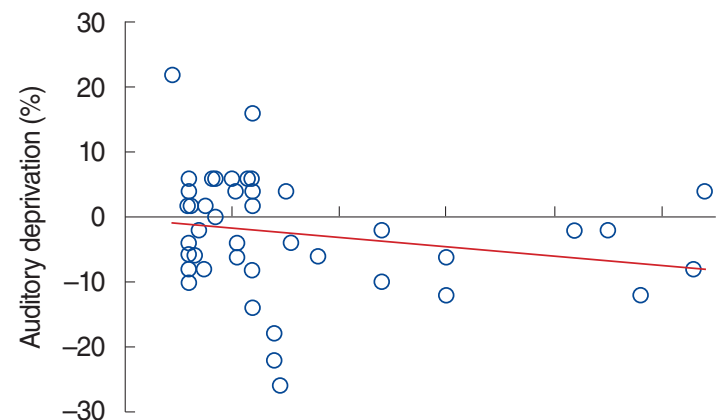

$$
\begin{array}{lrrrr}
20 & 40 & 60 & 80 & 100 \mathrm{~dB} \mathrm{HL} \\
& \text { Duration of HA use }(\mathrm{mo}) & \text { B }
\end{array}
$$

Fig. 3. Effects of the initial hearing threshold and duration of hearing aid (HA) use on auditory deprivation in the contralateral ears of patients with symmetric sensorineural hearing loss who had worn a unilateral HA for more than 1 year. Auditory deprivation was defined as the difference in changes in the word recognition score between the ipsilateral and contralateral ears. (A) The initial hearing threshold in PTA 4 shows a significant negative association with auditory deprivation. Auditory deprivation in the contralateral ear shows a negatively correlation with the initial hearing threshold in $\mathrm{PTA}_{4}\left(y=-0.3759 \chi+17.770, R^{2}=0.266, P<0.001, \chi=\right.$ initial threshold). (B) The duration of HA use does not have an effect on the severity of auditory deprivation in the unaided ear $\left(y=-0.069 \chi-4.765, R^{2}=0.0412, P=0.18, \chi=\right.$ duration of $\mathrm{HA}$ use). PTA , the average threshold at 500, 1,000, 2,000, and 4,000 Hz. 
threshold at $\mathrm{PTA}_{4}$ deteriorated. However, the duration of HA use was not significantly correlated with auditory deprivation in the contralateral ear though auditory deprivation tended to be more severe in the patients with longer use of HA (Fig. 3B).

\section{DISCUSSION}

In the present study, we identified auditory deprivation in the unaided ear in patients with symmetric SNHL wearing a unilateral HA. Notably, patients with a poor initial hearing threshold exhibited more severe auditory deprivation in the unaided ear. The concept of auditory deprivation has generally been accepted for a number of years. Several studies have reported auditory deprivation in the unaided ear of patients wearing a unilateral HA $[5,8,12,13]$. However, no study has reported the conditions that lead to increased susceptibility to severe auditory deprivation in patients with unilateral HAs. In addition, most previous studies have used analogue, linear type of HAs, rather than the more advanced digital, nonlinear type of HAs. It can be speculated that patients wearing unilateral HAs with more advanced auditory rehabilitation functions are likely to exhibit more severe auditory deprivation in the unaided ear.

In the present study, we analyzed the severity of auditory deprivation after unilateral insertion of nonlinear, digital HAs. The difference in $\triangle \mathrm{WRS}$ between the HA-aided (ipsilateral) and HAunaided (contralateral) ears was approximately $3 \%$ after $31.0 \pm$ 26.2 months from the date of HA insertion. Hurley [14] described that the prevalence of auditory deprivation at the unaided ear would be $8 \%$ at 3 years post fitting and $26 \%$ at 5 years post fitting. Although the duration of HA use were quite variable in the present study, nonlinear, digital types of HAs does not seem to have more severe unaided ear effect compared to the nonlinear, analogue types of HAs at the least. The author [14] also suggested a cutoff value for predicting auditory deprivation in a study involving 77 unilaterally and 65 bilaterally fitted adults. Auditory deprivation was observed in the unilaterally fitted individuals, with a mean initial pure-tone average of $46 \mathrm{~dB} \mathrm{HL}$ (3 frequency average based on $500,1,000$, and $2,000 \mathrm{~Hz}$ ), but not in the unilaterally fitted group, with a mean initial pure-tone average of $35 \mathrm{~dB} \mathrm{HL}$ [14]. In the present study, we also found that the group with $\mathrm{PTA}_{4} \geq 53 \mathrm{~dB}$ HL showed the more deteriorated auditory deprivation; several reasons such as different definition of auditory deprivation and type of word recognition test between two studies may be attributable to the difference of cutoff value. Therefore, it could be concluded that the amount of hearing loss plays a major role in the severity of auditory deprivation in digital, non-linear HAs as well as analogue, linear HAs.

Basically, bilateral HA fitting has been recommended for patients with severe-to-profound symmetric SNHL $[15,16]$. Byrne et al. [15] suggested that mildly impaired listeners, those fitted unilaterally performed as well, on average, as those fitted bilat- erally. However, Aided localization ability test results supported bilateral fitting for moderately and severely hearing-impaired listeners [16]. Even in the studies with children with severe SNHL, bilateral HAs were reported to be mandatory to prevent auditory deprivation $[12,17]$. The other study showed that a bilateral HA benefit was predominantly observed with respect to speech reception in noise, listening effort, and localization. This effect tended to be larger for the severe hearing loss patients than for the mildly hearing-impaired subjects [18]. Further, Boymans et al. [19] suggested that the patients with more severe hearing loss showed a higher bilateral benefit for the speech recognition test than did patients with milder hearing losses. However, several issues, such as cost, convenience, and stigma, hinder the acceptance of bilateral HAs by individuals with symmetric SNHL. If such drawbacks of bilateral HA fitting were serious issues for patients with symmetric SNHL, unilateral HA fitting can be alternative choice for hearing rehabilitation in the limited cases who have good initial hearing thresholds (i.e. less than $53 \mathrm{~dB}$ ). Nevertheless, we should enthusiastically encourage patients to get bilateral HAs because it becomes much more difficult to rehabilitate the auditory-deprived ear after unilateral HA fitting.

Meanwhile, WRS tends to be decreased even after wearing a HA in the aided ear. This may be associated with the feature of hearing loss over age. General aging of the auditory system seems to be the predominant factor. The relevant aging processes include damage to hair cells, loss of blood supply in the cochlea, and loss of nerve fibers in the central auditory system. Central auditory hearing loss results from both the general effects of biological aging and the effects of attenuation of neural input from ears that exhibit peripheral pathology [20]. This results the decreased speech understanding which is not improved by using HAs [21]. These may explain the cause of decreased WRS in the aided ear.

The present retrospective study has several limitations. This study reported patients' WRS with live-voice materials. Although the speech test was performed by a skilled audiologist, their use of live-voice materials may have been insensitive to a difference between ears for $\mathrm{PTA}_{4}<55 \mathrm{~dB}$ HL. In addition, relatively small size of samples could make a biased result that the duration of HA was not correlated with the severity of auditory deprivation, which was inconsistent with the previous studies. In the future study, case analysis with larger sample size is promising to get more concrete conclusions.

Unilateral HA fitting induces auditory deprivation in the unaided ear in patients with symmetric SNHL. Because auditory deprivation tends to occur easily in patients with severe-to-profound SNHL, bilateral HA insertion should be considered on the basis of the initial hearing function to prevent auditory deprivation in the unaided ear. 


\section{CONFLICT OF INTEREST}

No potential conflict of interest relevant to this article was reported.

\section{ACKNOWLEDGMENTS}

This work was supported by the Korea Health Technology R\&D Project, Ministry of Health \& Welfare, Republic of Korea (HI16C0142 to JJ).

The authors acknowledge the participation of the research subjects.

\section{ORCID}

Hyun Jin Lee $\quad$ https://orcid.org/0000-0003-3826-8830

Jeon Mi Lee https://orcid.org/0000-0003-2793-9045

Gina Na https://orcid.org/0000-0001-8111-2124

Young Min Moon https://orcid.org/0000-0002-3871-5242

Chan Lee https://orcid.org/0000-0003-0365-757X

Jinsei Jung https://orcid.org/0000-0003-1906-6969

\section{AUTHOR CONTRIBUTIONS}

Conceptualization: JJ. Data curation:YMM. Funding acquisition: JJ. Formal analysis: CL. Methodology: GN. Project administration: HJL.Visualization: JJ.Writing - original draft: HJL.Writing review \& editing: JML, JJ.

\section{REFERENCES}

1. Cruickshanks KJ,Wiley TL, TweedTS, Klein BE, Klein R, Mares-Perlman JA, et al. Prevalence of hearing loss in older adults in Beaver Dam, Wisconsin. The Epidemiology of Hearing Loss Study. Am J Epidemiol. 1998 Nov;148(9):879-86.

2. Johnson CE, Danhauer JL, Ellis BB, Jilla AM. Hearing aid benefit in patients with mild sensorineural hearing loss: a systematic review. J Am Acad Audiol. 2016 Apr;27(4):293-310.

3. Arlinger S. Negative consequences of uncorrected hearing loss: a review. Int J Audiol. 2003 Jul;42 Suppl 2:2S17-20.
4. Silverman CA, Emmer MB. Auditory deprivation and recovery in adults with asymmetric sensorineural hearing impairment. J Am Acad Audiol. 1993 Sep;4(5):338-46.

5. Silman S, Gelfand SA, Silverman CA. Late-onset auditory deprivation: effects of monaural versus binaural hearing aids. J Acoust Soc Am. 1984 Nov;76(5):1357-62.

6. Silman S, Silverman CA, Emmer MB, Gelfand SA.Adult-onset auditory deprivation. JAm Acad Audiol. 1992 Nov;3(6):390-6.

7. Martin FN, Forbis NK. The present status of audiometric practice: a follow-up study. ASHA. 1978 Jul;20(7):531-41.

8. Silverman CA, Silman S, Emmer MB, Schoepflin JR, Lutolf JJ. Auditory deprivation in adults with asymmetric, sensorineural hearing impairment. J Am Acad Audiol. 2006 Nov-Dec;17(10):747-62.

9. Murray DJ, Hanson JV. Application of digital signal processing to hearing aids: a critical survey. J Am Acad Audiol. 1992 Mar;3(2): 145-52.

10. Keidser G, Dillon H, Flax M, Ching T, Brewer S. The NAL-NL2 prescription procedure. Audiol Res. 2011 Mar;1(1):e24.

11. Han TH, Park CI.A study on standardization of Korean PB word list for speech audiometry. Korean J Otorhinolaryngol Head Neck Surg. 1981;24(3):265-72.

12. Gelfand SA, Silman S. Apparent auditory deprivation in children: implications of monaural versus binaural amplification. J Am Acad Audiol. 1993 Sep;4(5):313-8.

13. Gelfand SA, Silman S, Ross L. Long-term effects of monaural, binaural and no amplification in subjects with bilateral hearing loss. Scand Audiol. 1987;16(4):201-7.

14. Hurley RM. Onset of auditory deprivation. J Am Acad Audiol. 1999 Nov-Dec;10(10):529-34.

15. Byrne D, Noble W, LePage B. Effects of long-term bilateral and unilateral fitting of different hearing aid types on the ability to locate sounds. JAm Acad Audiol. 1992 Nov;3(6):369-82.

16. Gatehouse $S$. The time course and magnitude of perceptual acclimatization to frequency responses: evidence from monaural fitting of hearing aids. J Acoust Soc Am. 1992 Sep;92(3):1258-68.

17. Hattori H. Ear dominance for nonsense-syllable recognition ability in sensorineural hearing-impaired children: monaural versus binaural amplification. J Am Acad Audiol. 1993 Sep;4(5):319-30.

18. van Schoonhoven J, Schulte M, Boymans M,Wagener KC, Dreschler WA, Kollmeier B. Selecting appropriate tests to assess the benefits of bilateral amplification with hearing aids. Trends Hear. 2016 Jul; 20:2331216516658239.

19. Boymans M, Goverts ST, Kramer SE, Festen JM, Dreschler WA. A prospective multi-centre study of the benefits of bilateral hearing aids. Ear Hear. 2008 Dec;29(6):930-41.

20. Chisolm TH,Willott JF, Lister JJ.The aging auditory system: anatomic and physiologic changes and implications for rehabilitation. Int $\mathrm{J} \mathrm{Au-}$ diol. 2003 Jul;42 Suppl 2:2S3-10.

21. Bentler RA, Niebuhr DP, Getta JP,Anderson CV. Longitudinal study of hearing aid effectiveness. I: objective measures. J Speech Hear Res. 1993 Aug;36(4):808-19. 